\title{
Releasing of The White Paper on Medical Imaging Artificial Intelligence in China
}

\author{
Chinese Innovative Alliance of Industry, Education, Research and \\ Application of Artificial Intelligence for Medical Imaging
}

Key words: white paper; medical imaging; artificial intelligence

OSID code

I $\mathrm{N}$ the afternoon of March 26, 2019, The White Paper on Medical Imaging Artificial Intelligence in China (hereinafter referred to as the "white paper") was officially released in Beijing by the Chinese Innovative Alliance of Industry, Education, Research and Application of Artificial Intelligence for Medical Imaging (CAIERA) (Figure 1). The white paper was co-operatively written by the medical imaging experts from the tertiary Chinese hospitals, the scientific experts from AI research institutions and the leading AI medical enterprises in China. The contents of the white paper not only cover the upto-date application of AI in medical field, the latest advances of AI algorithms in medical image processing, the data requirement for medical AI development, and the current situation of structured data, but also expatiate the goal and challenge of clinical application for medical imaging AI development in 16 medical subject areas, which helps to identify the demands and opportunities for the AI industry. Forty representative enterprises of AI medical imaging in

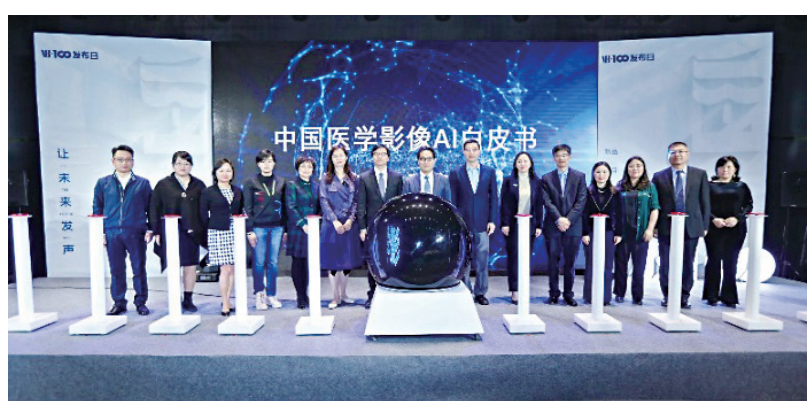

Figure 1. A group Photo of the initiators and cooperators (partial) in the launch event on March 26, 2019 in Beijing.
China were introduced in the white paper. The white paper points out that there are three key problems to be solved in development of AI products: the robustness, ease of usage and data security, which provides guidance of direction and strategy for the enterprises. Particularly, in view of the national policy on developing AI, the white paper gives a profound analysis on the challenges and the opportunities that medical imaging AI is facing. These contents agglomerate the cutting-edge efforts of experts in the industry-academia-research-application chain of medical AI, represent the mainstream voice of the society in China. The white paper teases out the applications of AI in medical imaging and analyses the current status and the future trend of the industry. It helps the related stakeholder to better understand policy trend and opportunities, and thus to explore appropriate strategy for further development. Importantly, it will play a guiding role in understanding market demands and establishing standardized systems in the path of landing AI products in the field medical imaging. Full text is publicly accessable from the CAIERA website, or by scanning the 2D code as in Figure 2.

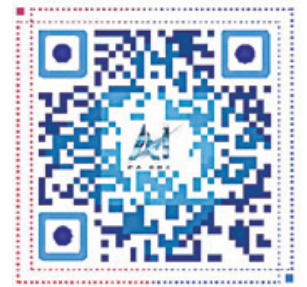

Figure 2. The $2 \mathrm{D}$ code for mobile reading of the full text of the white paper. 